\title{
El espesor del camino, visto a través de dos proyectos de transporte en Argelia
}

Aniss M. Mezoued

Escuela Politécnica Federal de Lausana, Suiza / Facultad de Arquitectura, Ingeniería Arquitectónica y Planificación Urbana, Universidad Católica de Lovaina, Bélgica.

\section{Khaled Amcha}

Facultad de Arquitectura, Ingeniería Arquitectónica y Planificación Urbana, Universidad Católica de Lovaina, Bélgica.

\author{
Bernard Declève \\ Facultad de Arquitectura, Ingeniería Arquitectónica y Planificación Urbana, Universidad \\ Católica de Lovaina, Bélgica.
}

Recibido: 4 de septiembre de 2017. Aceptado: 22 de diciembre de 2017.

\section{Resumen}

La planificación y la ingeniería de la movilidad han considerado al camino como una simple línea continua que conecta un punto A con un punto B. En términos de transporte, estos puntos son concebidos como lugares situados en el espacio-tiempo, mientras que su conexión sería un no-lugar que es necesario reducir en tiempo de recorrido y/o distancia. Esta representación, relacionada con la movilidad, no refleja la realidad del camino ni su espesor territorial. En cambio, él implica a una multiplicidad de actividades, temporalidades y representaciones que se superponen, como un palimpsesto, y lo hacen un lugar como cualquier otro. Entonces, la línea es solo una representación que no refleja la realidad, ya que ésta incluye porciones del territorio a ambos lados del camino. El artículo contribuye primero distinguir entre espesor vertical y horizontal. Luego, explora algunos métodos de identificación y caracterización del espesor, que toman a la escala local y al caminar como referencias, y que muestran la dificultad de delimitarlo en diferentes escalas. El texto se articula a través de dos casos en Argelia, que muestran cómo la planificación del transporte, a escala nacional y regional, niega este espesor territorial y perturba los sistemas de caminos a escala local.

Palabras clave

\section{Espesor} Camino Argelia

Tranvía

Autopista

Palavra-chave

Espessura Caminho

Argélia

Bonde

Autoestrada 


\title{
Lane and pathway thickness: definition essay based on two case studies of algerian transport projects
}

\begin{abstract}
Planning and transport engineering have for a long time considered pathways and lanes as continuous lines connecting a point $\mathrm{A}$ to a point $\mathrm{B}$. In terms of transport, those points are considered as places located in the space-time, while the link between them is considered as a "no-place" that should be reduced in terms of travel time or distance. This transport and mobility representation does not take into consideration the reality of pathways' territorial thickness. The latter relates on the first hand to the multiplicity of activities, temporalities and representations that are overlaid on each other like a palimpsest. On the other hand, thinking in terms of lines is quite reductive. It denies the fact that a line has a thickness that includes a part of the crossed territory on both sides of the line itself. This paper will contribute first to define the territorial thickness of pathways or lanes. It will distinguish between vertical and horizontal thickness. Then, the text will explore methods to identify and characterize it. Finally, through two case studies in Algeria, we will show how transport planning at local as well as regional scales denies this thickness and disturbs lanes and pathways systems.
\end{abstract}

\section{Introducción}

En los mapas, los caminos generalmente están representados por líneas. El conjunto de líneas conforma tanto una estructura espacial como un objeto territorial específico: le red vial. Dentro de la lógica de los ingenieros del transporte, la red toma consistencia autónoma, más allá de las relaciones que logre o no instalar entre los puntos en el espacio y con las áreas vecinas. De hecho, cada línea corresponde a una tecnología específica que contempla tanto el proceso de construcción, gestión y mantenimiento de la infraestructura física-que permite el desplazamiento- como el conjunto de técnicas al servicio de los vehículos o de los viajeros. Esas tecnologías están relacionadas con una "métrica", es decir: con una forma de medir la relación de los modos de desplazamiento con el territorio (Levy, 2006).

En el régimen territorial globalizado, la jerarquía que se establece entre las vías como líneas y su tecnología, prioriza las métricas que dan mayor importancia a la velocidad. De esa manera, las líneas y redes que se consideran más relevantes son aquellas cuyos usuarios pueden desplazarse con mayor velocidad y llegar más lejos, sin tener que sufrir las "rugosidades del territorio" (para retomar el término propuesto por Milton Santos, 1990). Un buen trazo, en esa perspectiva, es el que logra liberarse del tiempo y del espacio gracias a la velocidad, disminuyendo al máximo su adherencia al territorio (Amar, 1993; Lévy, 2005; y Mezoued, 2015 y 2016) y su relación con el patrimonio territorial sobre el que traza su trayectoria. Así, liberada de obstáculos, la vía determina paradójicamente un espacio-tiempo para cierta movilidad específica, pero al mismo tiempo establece un "no-lugar" (Augé, 1992), una ruptura con los usos y las estructuras del espacio atravesado.

Este artículo busca plantear esta problemática en una perspectiva crítica, basada en la consideración de las rupturas espaciales múltiples producidas por ese tipo de sistema, para proponer una revaloración de las performances que se producen desde las vías. Así, se prioriza la lectura de sus articulaciones potenciales con el espacio local, más que su performance en términos de adecuación a las exigencias

Keywords

Thinkness Path Algeria Tramway Highway 
de la velocidad. Nuestra perspectiva explora las relaciones entre urbanismo, territorios y movilidad. Por un lado, esta aproximación quiere subrayar -en el buen sentido- concepciones como la de las "sendas de mulas", de menor aceleración y mayor adherencia; por otro lado, más allá de la capacidad de generar conectividad longitudinal (es decir, de vincular los puntos de un territorio), se busca definir las relaciones territoriales transversales, a partir de lo que proponemos llamar el "espesor del camino".

Exploramos esta problemática en un contexto particular: Argelia, un país de 40 millones de habitantes, con una superficie de 2,5 millones de kilómetros cuadrados. Después de la crisis económica que atraviesa en la década de 1980, y luego de la crisis política y de seguridad que acompañó a la guerra civil de la década de 1990, Argelia retoma su estabilidad y su prosperidad a partir del año 2000. La renta petrolera y gasífera, asociada a los precios elevados de los hidrocarburos, permite que el país desarrolle ciertos megaproyectos, algunos de ellos específicamente vinculados al transporte. Se realizan diversas infraestructuras en el conjunto del territorio nacional: autopistas, vías férreas, metros, tranvías y teleféricos, entre otros. Esos proyectos tienen en común su concepción centrada en la ingeniería del transporte, sin una articulación con el desarrollo urbano ni territorial. Así se desencadena una serie de problemáticas a diferentes escalas. Para enfrentarlas sugerimos este trabajo sobre el espesor del camino, como pista para mejorar el despliegue de las infraestructuras en el territorio.

\section{El espesor del camino como perspectiva teórica}

Con la noción de espesor territorial -y considerando las perspectivas abiertas por varios autores- quisiéramos contribuir al análisis crítico de las condiciones de integración del espacio y el movimiento en un régimen territorial local. La construcción de un camino da lugar a trasformaciones territoriales múltiples: materiales, económicas, institucionales, culturales y de sociabilidad (Amar, 1993; Clot-Goudard y Tillous, 2008; Declève, 2015; Joseph, 1984). Al sobreponerse en el espacio como si fuese un palimpsesto (Corboz, 2001), esas trasformaciones multiplican tanto las "rugosidades territoriales" (Santos, 1990) como las posibilidades de generación de procesos territoriales inéditos. Al mismo tiempo contribuyen a la evolución de un "horizonte de sentido" (Corboz, 2001) asociado a cierto "proyecto territorial".

En la práctica, la noción de espesor del camino se refiere a una concentración de construcciones, personas y actividades, así como a flujos de intercambios de bienes y significados en torno del y por el camino. En ese sentido constituye una condición para la generación y la existencia de la intensidad urbana, es decir: de la carga de producciones y consumos del territorio por unidad de tiempo (Declève, 2009). La intensidad urbana es una variable determinante de la calidad de vida en la ciudad y, en el transcurso del tiempo, esa carga integra componentes simbólicos (Dekkil, 2009) que le dan más espesor al camino. Así, la vía toma consistencia y significado en la vida de las personas, tanto porque la atraviesan en lo cotidiano como en el tiempo más largo de la historia del territorio.

Cabe reconocer que la noción de espesor del camino todavía no está consolidada. En el sentido en el que la proponemos, no cuenta con una literatura abundante. Las referencias disponibles son generalmente descripciones relacionadas con casos particulares o con cierto tipo de infraestructura de transporte. En esos casos, el espesor del trazo parece variar según las escalas espaciales y temporales, los tipos de proyectos y las métricas involucradas. 
Sin embargo, es posible encontrar referencias útiles desde disciplinas alejadas de la planificación territorial y urbana o de la ingeniería del transporte. Este es el caso específico del término corredor, recuperado de la literatura del paisajismo y de la ecología (Forman, 2014). Los corredores están definidos como un elemento paisajístico lineal que permite el mantenimiento y la propagación de los espacios animales o vegetales entre dos hábitats, en el seno de un ambiente más o menos hostil. Puede ser largo o estrecho, recto o curvo. Los corredores son hábitats para unas especies mientras se plantean como barreras infranqueables para otras. Así, la noción permite hacer referencia a un ecosistema linear y continuo. Por eso, implica definir el caso del camino como un corredor que comprendería un conjunto de elementos vinculados unos con otros y sobre todo en relación directa con el camino en sí mismo. En ese marco, el corredor es usado frecuentemente para describir los bordes de las infraestructuras carreteras o de las autopistas y definir un perímetro de acción territorial alrededor de su unidad morfológica y/o funcional. Sin embargo, aunque la noción es utilizada en algunas vías, los planteamientos no definen con claridad el corredor o lo hacen en función de ciertos contextos. Es más, el término es utilizado en informes (Tecsult International Limitée, 2008) o en estudios de proyectos y no así en la literatura científica.

En el campo de los transportes carreteros y de autopista, la noción de área de influencia sin duda es la que se aproxima mejor a lo que denominamos espesor. Se trata de una discusión de desarrollo económico y a veces social. Su objetivo es definir una zona de impacto económico sobre el territorio (Berion y Feve, 2005). A más extendida sea su influencia, el impacto sobre el desarrollo económico será más importante (Fève, 1978; Plassard, 1977; y Varlet, 1997). La noción de área de influencia es independiente de la morfología y concierne sobre todo a una zona funcional. Si bien ésta puede resultar útil en la definición de espesor horizontal del camino, no es suficiente para afinar esa definición y tener precisión a diferentes escalas del territorio.

De hecho, el corredor y el área de influencia son pertinentes cuando se refieren a estructuradores lineares de grandes territorios, como una vía fluvial, férrea, carretera o a una autopista. Pero son menos pertinentes cuando se trata de aplicarlas a una escala urbana y a caminos menos largos y más pequeños, o cuando se trata de trabajar en un tejido territorial más fino. Así, no permiten una mirada integral de los anclajes de los caminos en los territorios.

Con esas referencias, este artículo busca contribuir a la consolidación conceptual de la noción del espesor. Para eso, proponemos un marco de lectura y análisis que permita aproximaciones comparativas e independientes de las especificidades de cada métrica. Abordamos, entonces, dos estudios de caso relativos a proyectos de grandes infraestructuras en Argelia: el primero es el de una línea de tranvía en Argel y el segundo es el de una nueva autopista que cruza el país de Este a Oeste. Pondremos en evidencia varias actividades, temporalidades, representaciones y escalas de proyectos que se sobreponen en la práctica de esos caminos y que contribuyen -o no- a integrarlos en la "masa territorial" (Magnaghi, 2003). En una segunda etapa, mostraremos cómo las infraestructuras contempladas producen su espesor, conectando partes del espacio y contribuyendo en la intensificación del sistema territorial. Los resultados precisamente permiten retomar la definición del espesor del camino. Así, sugerimos que sus dimensiones espaciales pueden ser traducidas en criterios de descripción y de diseño de las infraestructuras de movilidad, en términos de métodos de proyecto territorial.

En este sentido, la descripción destacará dos tipos de espesor. El primero, que nosotros proponemos llamar longitudinal, se refiere a todo aquello que se inscribe en el espesor de la línea trazada en los mapas de los geógrafos y en los planos de los ingenieros. Se trata de los espacios, infraestructuras, equipamientos, usos y servicios que componen la línea mediante la afectación física al terreno de ocupación de la infraestructura (nivel 


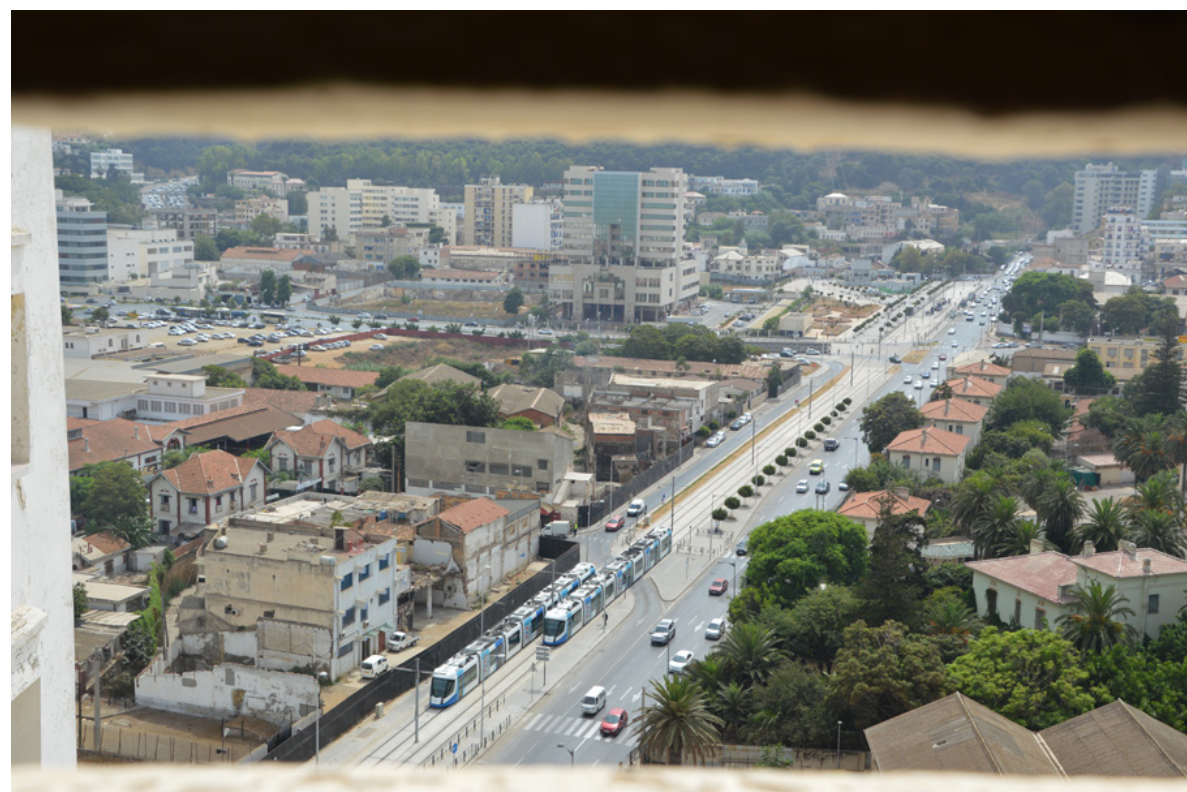

1: ocupación) o del espacio comprendido dentro de los límites de la zona de propiedad pública (nivel 2: propiedad pública). El segundo tipo de espesor, que denominaremos espesor transversal, tiene que ver con lo que sucede en los bordes inmediatos de la zona de propiedad pública (nivel 3: espesor colindante) o en el área de influencia de la infraestructura (nivel 4: espesor ampliado).

\section{Dos caminos, dos métricas y dos escalas territoriales}

El primer camino que nos interesa estudiar es el del tranvía de Argel. Su funcionamiento data del 2011, con una línea de más de $23 \mathrm{~km}$ que atraviesa varias comunas y barrios de la capital. Articula el centro histórico y la periferia residencial, pasando por zonas industriales y agrícolas. Así, el tranvía se presenta como un vínculo entre los fragmentos dispersos del territorio urbano argelino. Su planteamiento fue presentado para reducir el tráfico automovilístico y permitir una vinculación efectiva entre los lugares de residencia y de empleo. Sin embargo, el proyecto fue concebido y realizado con una lógica sectorial de transporte y sin tomar en cuenta las dinámicas del territorio atravesado. Por ejemplo, el espacio público fue intervenido dentro del espesor de las calles atravesadas por las líneas, pero sin afectar los límites de los predios construidos ni los terrenos privados (Figura 1).

El segundo camino es la autopista Este-Oeste. Con $1216 \mathrm{~km}$ de largo, es uno de los proyectos de infraestructuras de movilidad más grandes desarrollados en la historia de Argelia. Es parte de las prioridades del plan director carretero nacional (SDRN 20052025) y del plan nacional de ordenamiento del territorio (SNAT 2025). En ambos planes representa uno de los ejes estructurantes de los proyectos de desarrollo económico y territorial. La autopista integra las principales ciudades argelinas (Skikda, Constantina, Sétif, Argel, Chlef, Orán y Tlemecén) y se espera que constituya un tramo de la unión con los países del Magreb (sobre $7 \mathrm{mil} \mathrm{km}$ ) y con el conjunto de la costa mediterránea (Figura 2).

Los dos casos que proponemos implican no solamente modos de transporte diversos, sino también escalas de territorios completamente diferentes. En el primero estamos a una escala urbana, entre la conexión metropolitana y el desplazamiento local; la
Figura 1. Vista del tranvía de Argel y del espacio público intervenido en el espesor de la calle. Fuente: Mezoued, 2015. 


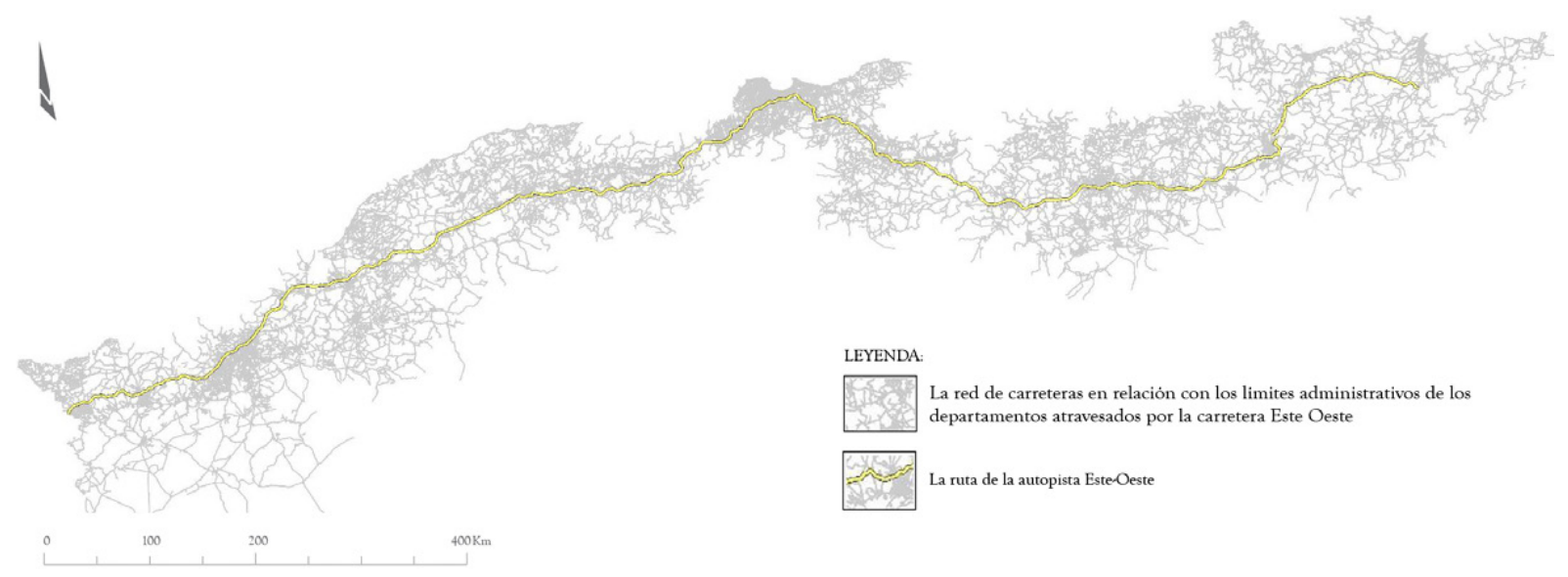

métrica peatonal es su referencia, ya que el usuario del tranvía es un peatón que hace una pausa (Amar, 1993; Lévy, 2005; Mezoued, 2015). En el segundo caso estamos en una escala nacional y de gran territorio. La métrica de referencia es la del automóvil, ya que asegura la vinculación sobre el territorio atravesado por la carretera (Bres, 2005). En ese sentido, la relación entre cada tipo de camino con el territorio es de naturaleza diferente. Por eso, la definición de espesor debe adaptarse a cada configuración y considerar el juego de escalas territoriales, el modo de funcionamiento de las métricas, el diseño de la infraestructura, la configuración y el rol de las pausas y de las paradas, etc.
Figura 2. Mapa de los $1216 \mathrm{~km}$ de la autopista Este-Oeste. Fuente: Amcha, 2016.

\section{Dos niveles de espesor longitudinal: ocupación y propiedad pública}

Incluimos como parte del espesor longitudinal todos aquellos elementos que se representan en el trazo diseñado en el mapa. Podemos considerar dos niveles: por un lado, los predios sobre los que el camino se instala físicamente (nivel 1: ocupación) y, por otro lado, los límites de la zona de propiedad pública (nivel 2: propiedad pública). En el caso de la línea de tranvía de Argel el primer nivel de espesor corresponde a los 7,5 m ocupados por los rieles, además del ancho de los andenes en las paradas. En el desarrollo práctico del proyecto urbano, la delimitación del ocupación -a priori considerado por los operadores de transporte como un inventario técnico no negociable- se constituye en objeto de múltiples debates entre los operadores de transporte que comparten el mismo espacio público. Esos debates pueden conducir a una modificación del trazo inicial previsto o a una modificación de los estándares técnicos normalmente adoptados. En Argel, por ejemplo, la implantación del tranvía ha supuesto una reducción de los predios destinados a las vías públicas situadas en el trazo de la nueva infraestructura.

La problemática de definición de la zona de propiedad pública en torno al proyecto es aún más compleja. Desde una posición jurídica, es posible considerar que el espesor del proyecto está delimitado por el terreno que pertenece a operador; sin embargo, también se puede considerar que el dominio público está asociado al proyecto que desborda los límites de la propiedad y se esparce hasta los bordes del espacio público con el que está relacionado. En el caso del tranvía de Argel, desde esta segunda perspectiva, el área de dominio del proyecto corresponde no sólo al espacio de influencia que atraviesa el proyecto, sino además a las plazas, los jardines y las aceras que bordean su trayecto (Figura 3 ).

En el caso de la autopista Este-Oeste, la equivalencia entre la zona de ocupación y de propiedad pública del proyecto se establece a priori. El espesor de propiedad pública está constituido por el derecho de vía, que corresponde a tres veces el ancho de la 


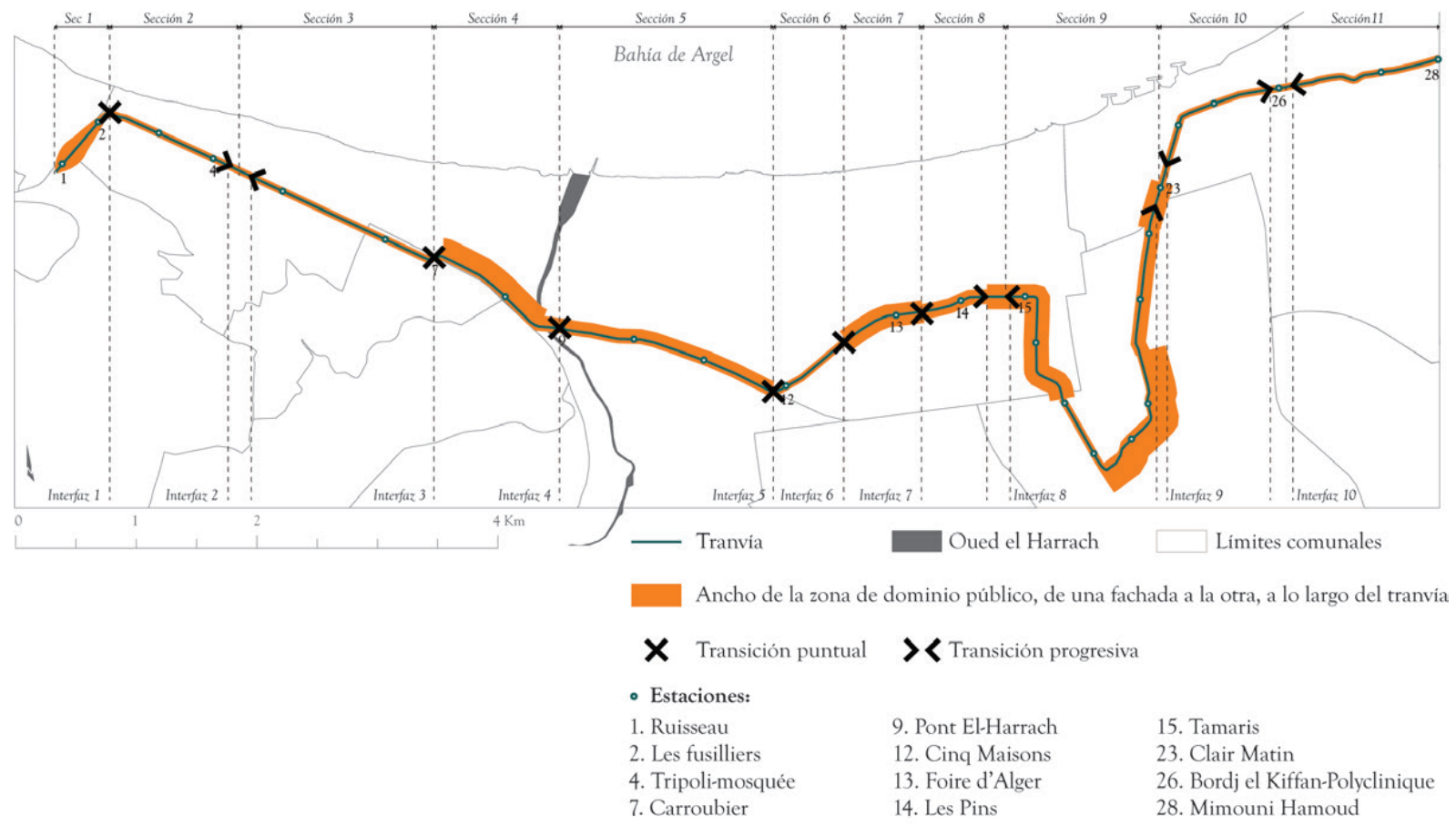

carretera más las calzadas y carriles de emergencia. Se define también por las estaciones de servicio, áreas de descanso e intercambiadores de carreteras. La estandarización de este espesor es mucho más elevada que la observada en el tranvía, ya que éste segundo debe adaptarse a las redes viales existentes. Otra diferencia es que el tranvía permite cierta permeabilidad de otros flujos a lo largo de su trazo, mientras que en la autopista no existe autorización para otro tipo de flujos que no sean los automóviles. La autopista funciona como un túnel o un tubo con puertas de entrada y salida hacia los territorios que irriga. Desde ese punto de vista, propicia un menor anclaje en el espacio que atraviesa.

\section{El espesor transversal colindante}

En lo referido al área de dominio, el espesor colindante alude los terrenos ubicados en ambos lados del camino, que conforman los bordes y que mantienen una relación funcional con el espacio del movimiento.

En el caso del tranvía, nosotros definimos e identificamos este espesor en los bordes vecinos. Se trata de una aproximación morfológica, que propone analizar el primer manzano, construido o no, que colinda con el camino. Después del lote o la parcela, el manzano constituye la primera unidad morfológica y funcional que está relacionada directamente con el tranvía. La configuración de los bordes juega un rol importante dentro de los anclajes del camino en el territorio inmediato. Si la infraestructura está en un predio privado, asegurado por barreras o por muros herméticos, su anclaje en el territorio es débil. $\mathrm{Si}$, al contrario, existe porosidad o la morfología construida permite un contacto directo con la línea de transporte, entonces su anclaje en el territorio es importante. En todo caso, el análisis de los bordes muestra lo que clasificamos en cuatro tipos (Figura 4).

Por una parte, están los bordes abiertos, que generalmente son los espacios sin construcción y muchas veces agrícolas; los lotes circundantes están constituidos por grandes

Figura 3. Espesor de la zona de propiedad y dominio público. Fuente: Mezoued, 2015. 


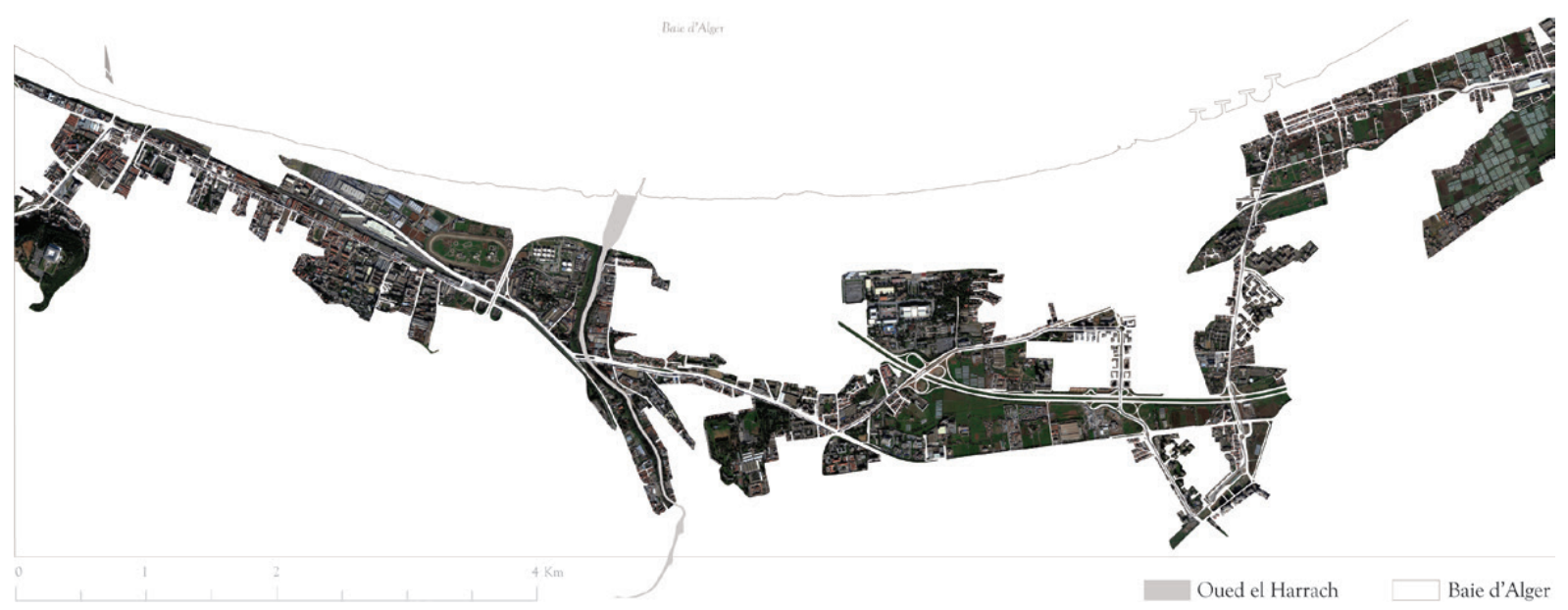

superficies de propiedad privada, que dan una sensación de apertura al trayecto del tranvía. Por otra parte, están los bordes permeables, que podemos encontrar a nivel de los grandes conjuntos cuyas construcciones están dispuestas de forma dispersa sobre grandes superficies de terreno. No existe un alineamiento continuo de edificios a lo largo de la acera y el espacio público penetra entre los bloques. Asimismo, están los bordes cerrados, que encontramos en diferentes lugares a lo largo de viaje sobre la línea; generalmente son construcciones integradas al tejido urbano, pero que se aíslan detrás de muros, sin mostrar un frente edificado ni medianeras hacia la vía. Son construcciones que corresponden a diversos actores, privados y públicos, y producen bordes totalmente opacos en torno al tranvía. No ofrecen permeabilidad, ni contacto con los edificios ni con los espacios públicos; se podría afirmar que muestran muy poco de la "urbanidad" como tal. Finalmente, están los bordes con edificios construidos, que se encuentran principalmente en los centros históricos. Este tipo de malla urbana teje un espesor que favorece la métrica peatonal, al igual que el contacto con el camino (Mezoued, 2015).

Un análisis más profundo de la ocupación del espacio de las prácticas a lo largo de la línea muestra que ciertas configuraciones permiten una intensificación urbana y que éstas espesan transversalmente el camino. Así se fabrican lugares y "urbanidad”. Se trata de bordes con frentes edificados donde el contacto directo con el espacio público parece favorecer las interacciones sociales (Figura 5) (Mezoued, 2015).

En el caso de la autopista, la delimitación de un espesor colindante es menos evidente. Existen algunos indicios que permiten la identificación de los bordes de las propiedades o de los manzanos, pero no existen mayores precisiones que tengan vinculación con la escala territorial alcanzada por la vía ni en función de la lógica de la métrica automóvil que moviliza. Es necesario sobrepasar el límite de esas primeras manzanas y pensar la relación con el territorio de diferente manera. La autopista frecuentemente es concebida como un túnel seguro, que se aísla del entorno inmediato, pese a que contribuye a la perspectiva de la aglomeración y a la valorización del suelo que se beneficia con el crecimiento urbano.

Por otra parte, la autopista atrae inversiones, gracias a la facilidad de desplazamiento de los bienes, de las personas y de las mercancías, así como al acceso a los territorios cuyo valor del suelo es menos elevado que en la ciudad (Philbrick, 1961) e incrementa la oferta de terrenos cerca a los intercambiadores de autopista. Estos últimos se convierten en puntos de concentración de nuevos asentamientos urbanos, constituyendo verdaderos espacios de intercambio de mercaderías, ideas y personas (Muller, 1979). Sobre esos puntos de intercambio o de conexión sugerimos que es posible identificar un espesor
Figura 4. El espesor de la zona de propiedad del tranvía Argelia. Fuente: Mezoued, 2015. 


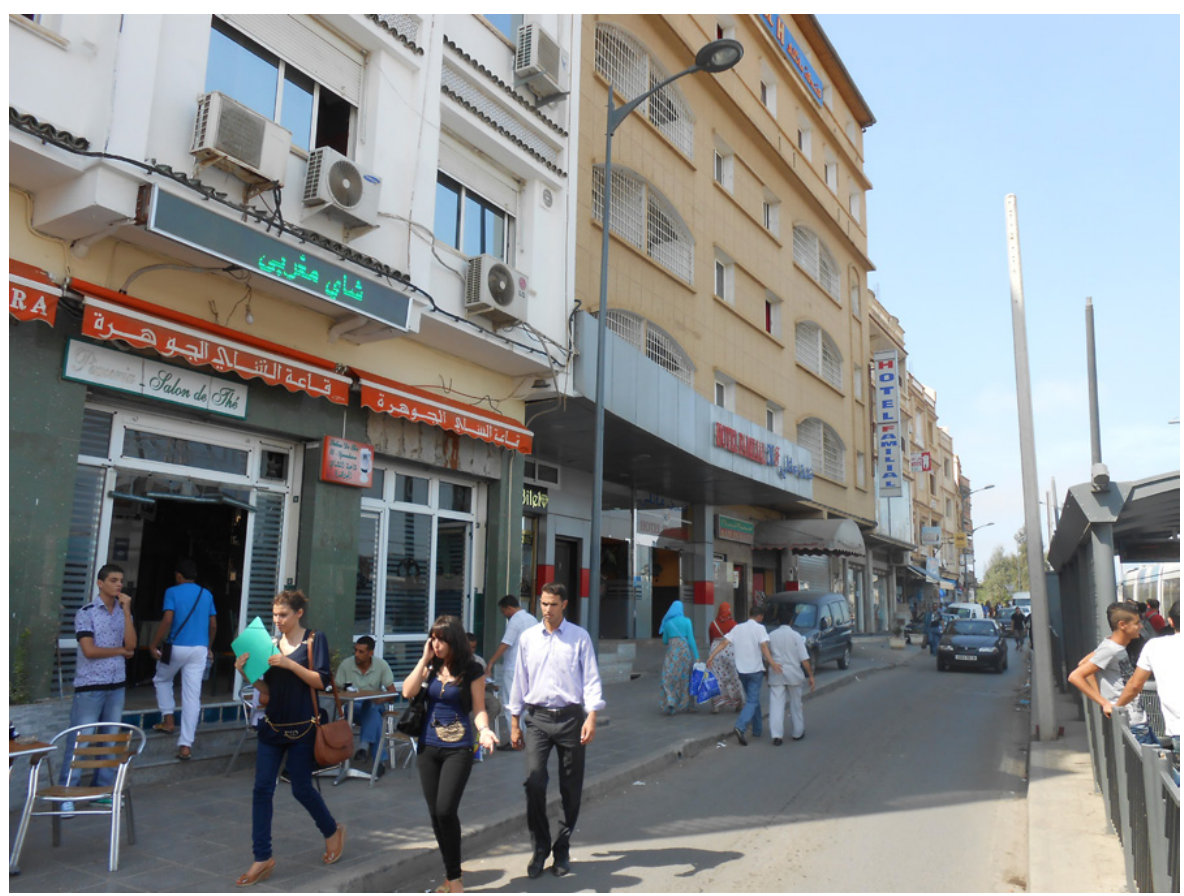

de colindancia. Para ayudarnos en este ejercicio, acudimos a la noción de colindancia (riverainité) características de los rieles. Ésta fue introducida por Marcelle Démorgon dentro de su análisis de la evolución de las vías nacionales y su conceptualización ha sido luego precisada por Antoine Brès (po2005). Este último define la colindancia a una vía como "le degré d'inscription sur ses rives des pratiques de mobilité quelle accueille" y se mide respecto a los servicios y las comodidades ofrecidos sobre los bordes. Brès precisa que, dentro de las vías, uno encuentra dos extremos: por una parte, la autopista con colindancia nula, que no tienen relación alguna con su entorno (pese a que el ordenamiento territorial muchas veces implica áreas de influencia de las carreteras regionales); por otra parte, está la calle continua de proximidad, que se asocia al espacio y al movimiento con lugares de descanso y con múltiples posibilidades de paradas en sus bordes.

En el caso de la autopista Este-Oeste de Argelia, es posible registrar, alrededor de los intercambiadores, a poblaciones, asentamientos o partes de las aglomeraciones que están morfológicamente y funcionalmente relacionadas con la autopista y que pueden ser denominadas como colindantes o vecinas. Sin embargo, la linealidad de la vía no ofrece a priori ningún espacio de colindancia. A nivel de la definición normativa de lo que se espera de una autopista, su espesor colindante puede ser considerado como nulo. Aun así, una mirada de mayor profundidad de algunas prácticas permite revelar algunos indicadores de cierto espesor colindante. Por ejemplo, sobre los bordes existe la presencia de puestos informales de venta de sándwiches o de legumbres que provienen de los campos vecinos (Figura 6). Las calzadas y los carriles de emergencia se transforman en lugares de pausas y descanso, pero también en ventanas abiertas que se enlazan con el territorio. Pese a ser lugares efímeros, las pausas crean una rugosidad dentro del espesor longitudinal de la línea, pero también le otorgan cierto espesor transversal y colindante en la medida en que dinamizan un potencial de interacción con el territorio de la autopista y el espacio rural del entorno.

Sin embargo, más allá de esas dinámicas, el espesor colindante implica también una dimensión poco analizada en lo referido a las infraestructuras carreteras: la relación causal y recíproca entre la infraestructura carretera y los aspectos ecológicos y paisajísticos (Forman et al, 2003). Se trata de una dimensión que esperamos profundizar en nuestras investigaciones.
Figura 5. Intensificación urbana a lo largo de los bordes construidos. Fuente: Mezoued, 2015. 


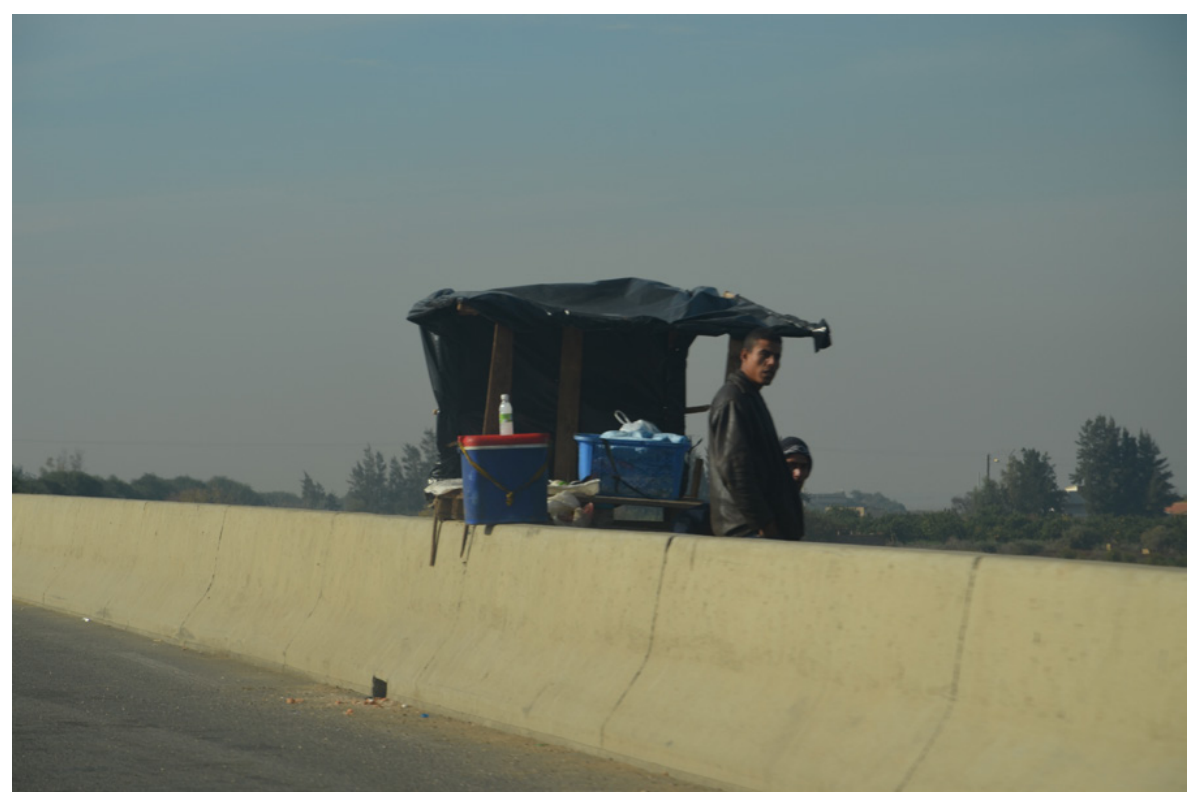

\section{El espesor transversal ampliado}

El último nivel de espesor es aún más extenso que los ya expuestos. Implica un territorio ampliado que comprende una red de caminos y de lugares que pueden estar relacionados con el camino principal, aunque no estén directamente conectados con él. El mejor ejemplo es el del concepto de "rutas del vino" en Francia. Se trata de un camino estructurante que atraviesa los viñedos vecinos, pero que conecta también una serie de lugares que participan en la actividad de producción, venta y degustación de los vinos. A veces se trata de lugares alejados entre sí, pero que constituyen un ecosistema económico y cultural. Eso se parece al área de influencia que nosotros señalamos anteriormente.

En el caso del tranvía, nosotros definimos este espesor respecto a la adherencia de la métrica. Es decir, su capacidad de multiplicar los contactos con el territorio que atraviesa y con los objetos y formas urbanas que constituyen ese territorio. Esa adherencia está garantizada por la multiplicación de puntos de anclaje (paradas), por una parte; y por el establecimiento de las relaciones entre los contactos dentro con el espesor del territorio, por otra parte. Eso se logra a través de la conexión a otras líneas cuando se trata de una escala grande, y a través del espacio público cuando se trata de la escala local (Mezoued, 2015 y 2016). En ese sentido, es posible comprender este espesor de dos maneras. Ya sea desde la lógica del transporte colectivo y a partir del conjunto de líneas que parten del tranvía para alcanzar un territorio más vasto dentro del tejido urbano metropolitano; o ya sea -como proponemos- al evidenciar la métrica peatonal como centro de nuestras preocupaciones. Consideramos que el camino representado por el tranvía debe permitir no solo seguir un viaje eficaz en términos lineales, sino además asegurar una continuidad del desplazamiento peatonal de una parte a la otra y sobre las huellas de su paso. Eso debe tomar en cuenta que la distancia cómoda para el desplazamiento peatonal es de 500 m aproximadamente, como define Leon Krier (1977), autor referente en la temática.

Con esa visión, un proyecto - entre movilidad y urbanismo- puede pasar por dos aproximaciones para el caso del tranvía. Primero podemos mencionar un abordaje metropolitano del espesor ampliado, que toma en cuenta el conjunto de la ciudad. O también hay una aproximación más bien local que se articula al espesor identificado con la distancia cómoda para el desplazamiento peatonal y la conexión de las calles y cominos locales (Figura 7).
Figura 6. Pausas informales. Fuente: Amcha, 2016. 


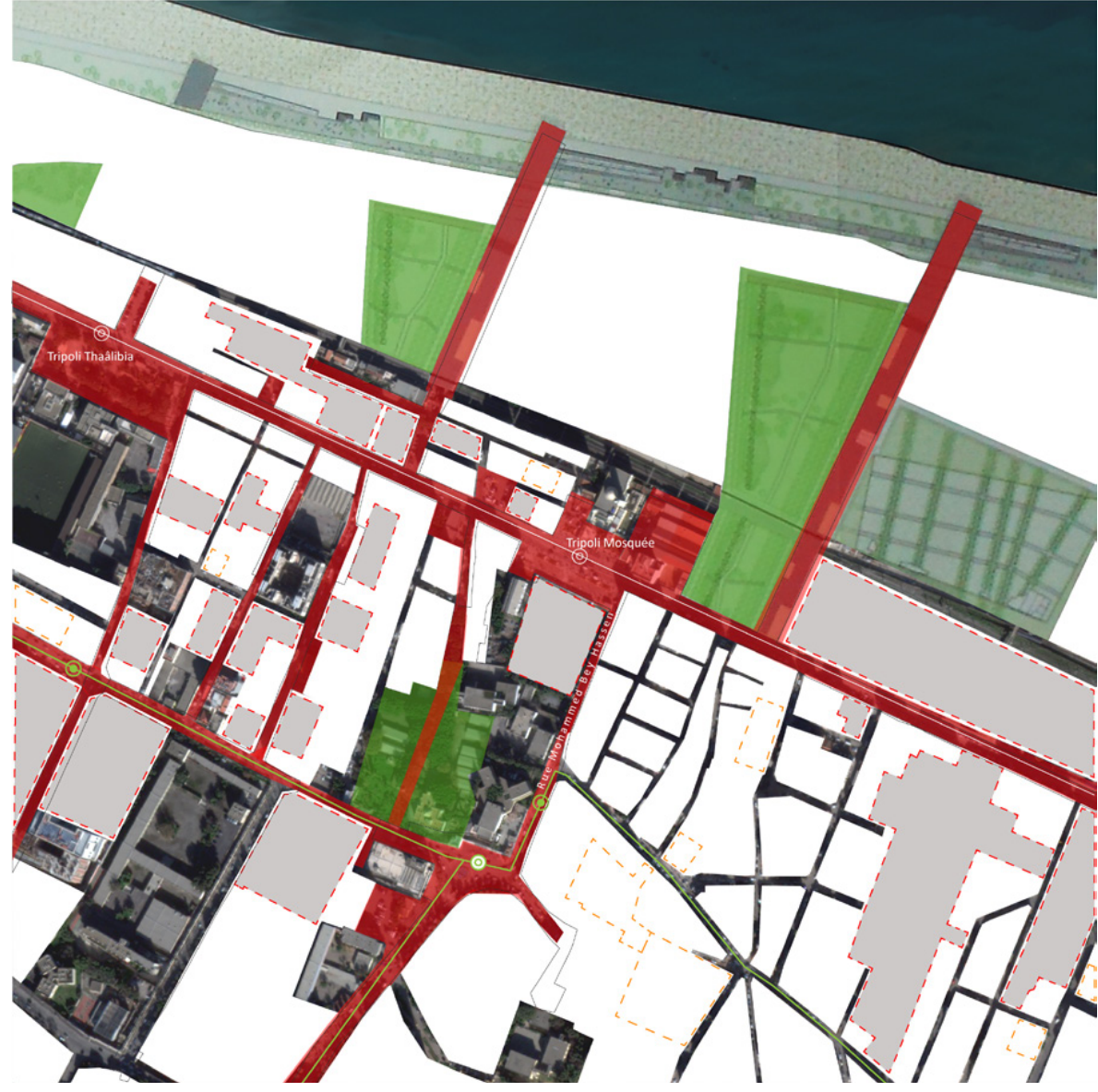

Para el caso de la autopista, el espesor ampliado está definido en la relación con un territorio mucho más grande, que puede ser el de la escala nacional. El área de influencia, entonces, es un término adecuado y preciso para comprender este tipo de espesor. De hecho, las ramificaciones de la autopista son las redes de vías rápidas que se distribuyen a partir de los intercambiadores y que permiten conectar los territorios distantes o las actividades económicas que están funcionalmente vinculadas a la carretera. Ese es el caso de el Puerto de Djendjen sobre la costa argelina (Figura 8). Se trata de un puerto comercial conectado a la autopista Este-Oeste por una carretera saliente de 110 kilómetros desde el intercambiador de El Eulma. Esta conexión fue pensada en relación directa con el sistema carretero y ferroviario. Así, el espacio entre el puerto y el intercambiador podría constituir una matriz del desarrollo de los territorios de la autopista que modifica su morfología y que se inscribe plenamente dentro del espesor de esta infraestructura.

La reflexión sobre el espesor de la autopista Este-Oeste en Argelia permite desarrollar la hipótesis que una carretera se caracteriza por un sentido de articulación de grandes territorios. Esa particularidad la distingue de otros tipos de caminos. Sus espesores inmediatos y la extensión son evolutivos y difusos. Por eso, son difíciles de espacializar y de ser delimitados de forma clara.

\section{Conclusión}

Esta modesta contribución a la definición de espesor del camino muestra la dificultad de aplicar de forma homogénea una misma matriz de análisis en la diversidad de caminos.
Figura 7. Proyecto de construc ción de un espesor ampliado. Fuente: Mezoued, 2015. 


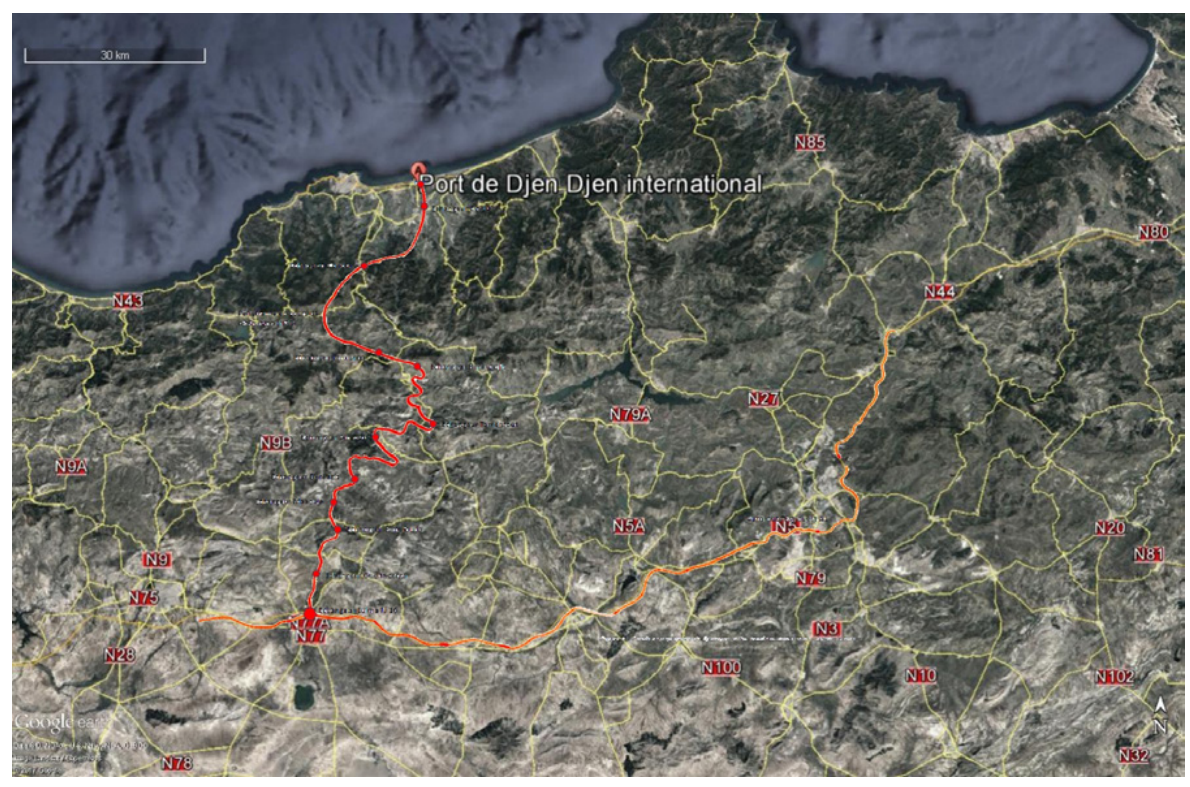

Cada uno, en función de su escala, de la métrica que utiliza y de su forma, construye su propio espesor y requiere de herramientas, aproximaciones y métodos específicos para definirla. En todo caso, eso es lo que nos muestra la lectura comparada de las dos infraestructuras de transporte propuestas como ejemplo en el artículo: el tranvía y la autopista. Sin embargo, de la misma forma que existen diferencias considerables, se mantiene la posibilidad de identificar los cuatro niveles de espesor propuestos. El ejercicio muestra que esas dimensiones sí dan cuenta de características compartidas, probablemente no sólo por los dos tipos de vías analizadas, sino para el conjunto de los caminos.

Finalmente, es importante comprender la cuestión del camino no como una simple línea sino como un espesor. Esto permite subrayar una complejidad de interacciones de movilidad, transporte y urbanismo. Así, se trata de un marco interpretativo que se muestra pertinente tanto para un análisis como para el desarrollo de proyectos territoriales.

La redacción de este artículo ha contado con la colaboración de Bianca De Marchi Moyano, en el cuidado de la traducción del francés al español.
Figura 8. Espesor ampliado en el caso del puerto de Djendjen. Fuente: Amcha, 2017. 


\section{Q Bibliografia}

》 AMAR, George (1993) Pour une écologie urbaine des transports. Les Annales de la Recherche Urbaine, 59-60.

" AUGE, Marc (1992) Non-lieux, introduction à une anthropologie de la surmodernité. Paris : Seuil.

» BERION, Pascal y FÈVE, Michel (2005) Les observatoires autoroutiers : du regard empirique aux apports scientifiques. Paper presented at the $7 \mathrm{e}$ rencontres de Théo Quant. http://thema.univ-fcomte.fr/theoq/pdf. resumes/TQ2005

» BRÈS, Antoine (2005) Inscription territoriale des mobilités et riveraineté des voies : faire halte aujourd'hui. Paris: Université Paris, Panthéon-Sorbonne.

»CLOT-GOUDARD, Rémi y TILLOUS, Marion (2008) L'espace du réseau : du flux au territoire. Le tournant pragmatiste engagé par Isaac Joseph. Tracés. Revue de Sciences humaines, 15 .

" CORBOZ, André (2001) Le territoire comme palimpseste et autres essais. Paris: Editions de l'imprimeur.

» DECLÈVE, Bernard (2009) Du ménagement de la nature à la naturalisation de la ville. Territoires Wallons, Vol II : 3, pp. 10-44.

»DEKKIL, Guillaume (2009) Introduction au numéro spécial de URBIA sur I'intensité urbaine. URBIA, 9, 3-12.

» FÈVE, Michel (1978) Impact économique des budgets routiers nationaux, régionaux et locaux. Revue générale des routes et des aérodromes, 548(77).

» FORMAN, Richard (2014) Urban Ecology. New York: Cambridge University Press.

» FORMAN, Richard et al. (2003) Road Ecology: Science and Solutions. Washington D.C.: Island Press.

» KRIER, Léon (1977) The city within the City. A+U (special issue), 69-152.

" LÉVY, Jacques (2005) Modèle de mobilité, modèle d'urbanité. In S. Allemand, F. Ascher, \& J. Lévy (Eds.), Les sens du mouvement : modernité et mobilités dans les sociétés urbaines contemporaines, pp. 157-169: Belin.

» MEZOUED, Aniss (2015) La mise en récit de l'urbanisme algérois, passé, présent, futur. A la recherche des conditions d'institution de l'espace public comme médiation et comme projet. Cas du tramway d'Alger. Louvain-la-Neuve: Presses universitaires de Louvain.

» MEZOUED, Aniss (2016) L'in-adhérence spatiale du tramway d'Alger à l'échelle locale. In M. Srir (Ed.), Dynamiques urbaines à Alger : la (re)fabrication de la ville en questions, pp. 247-265. Paris: L'Harmattan.

» MULLER, Thomas (1979) The Impact of Beltways on Central Business Districts - A Case Study of Richmond. Washington D.C: the Urban Institute.

" PHILBRICK, Allen (1961) Analysis of the geographical Patterns of Gross Land Uses and Changes in Numbers of Structures in Relation to Major Highways in the lower Half of the Lower Peninsula of Michigan. Michigan: Michigan State University.

»PLASSARD, François (1977) Les autoroutes et le développement régional. Lyon: Presses universitaires de Lyon. 
» SANTOS, Milton (1990) Espace et méthode. Paris: Publisud.

» TECSULT INTERNATIONAL LIMITÉE, e.-c. (2008) Elaboration du schéma d'aménagement du corridor de l'autoroute Est-ouest. Québec-Alger.

»VARLET, Jean (1997) Autoroutes, économies et territoires. Clermont-Ferrand: Ceramac.

» ZUNINO, Gwenäelle. (2009) Plaidoyer pour une ville intense. URBIA, 9, 57-76.

\section{Aniss M. Mezoued / aniss-mouad.mezoued@uclouvain.be}

Arquitecto urbanista y doctor en urbanismo. Ha desarrollado su tesis doctoral en la Universidad Católica de Lovaina (UCL, Bélgica) sobre el tranvía de Argel y luego un postdoctorado sobre la problemática de la ciudad lenta en la Escuela Politécnica Federal de Lausana (EPFL, Suiza). Actualmente es coordinador del Observatorio del Centro de Bruselas del Brussels Studies Institute y profesor invitado de la UCL. Sus investigaciones se centran en el espacio público, las formas urbanas y en la articulación entre movilidad y urbanismo.

\section{Khaled Amcha / khaled.amcha@student.uclouvain.be}

Arquitecto urbanista. Ha concluido su licenciatura en el Departamento de Arquitectura e Ingeniería Civil de la Universidad de Abdelhamid Ibn Badis de Mostaganem (Argelia) y su maestría de especialización en Urbanismo y Planificación Territorial en la Universidad Católica de Lovaina (UCL, Bélgica). Actualmente desarrolla una investigación doctoral sobre las relaciones entre movilidad-urbanismo y movilidad-territorio en torno a la autopista Este-Oeste en Argelia en la UCL.

\section{Bernard Declève / bernard.decleve@uclouvain.be}

Arquitecto ingeniero y urbanista. Profesor de la Facultad de Arquitectura, Ingeniería Arquitectónica y Planificación Urbana (LOCI), de la Universidad Católica de Lovaina (UCL, Bélgica). Sus investigaciones analizan el hábitat en las grandes ciudades y su influencia en los proyectos urbanos y territoriales, a partir de procesos colaborativos y participativos. Ha desarrollado experiencias internacionales en Europa, África y América Latina como experto en urbanismo participativo y en investigación-acción con actores públicos, empresariales, académicos y ONG. 